\title{
La quatrième tour de Hanoï
}

\author{
Thierry Bousch
}

\begin{abstract}
In the four-peg variant of the Towers of Hanoi game, it is well known that $N$ disks can be transferred from a column to another in $2^{\nabla 0}+2^{\nabla 1}+$ $\cdots+2^{\nabla(N-1)}$ moves, where $\nabla n$ denotes the largest integer $p$ such that $p(p+1) / 2 \leqslant n$, and it was conjectured that this number of moves was the minimum possible. We shall see, in this article, that is is indeed the case.
\end{abstract}

\section{Résumé}

Dans la variante à quatre colonnes des Tours de Hanoï, on sait bien qu'on peut transférer $N$ disques d'une colonne vers une autre en $2^{\nabla 0}+2^{\nabla 1}+$ $\cdots+2^{\nabla(N-1)}$ mouvements, où $\nabla n$ désigne le plus grand entier $p$ tel que $p(p+1) / 2 \leqslant n$, et on conjecturait que ce nombre de mouvements était le minimum possible. Nous verrons, dans cet article, que c'est effectivement le cas.

\section{Présentation du problème}

Le jeu des Tours de Hanö̈, inventé par le mathématicien français Edouard Lucas en 1883, a inspiré de nombreuses variantes. Une des plus intéressantes, The Reve's Puzzle, est donnée par le puzzliste anglais Henry Ernest Dudeney en 1908 dans son recueil de jeux mathématiques The Canterbury Puzzles [Dud]: on dispose d'un plateau muni de quatre aiguilles, et $N$ disques de taille strictement décroissante enfilés initialement sur une de ces aiguilles; le problème consiste à transférer tous ces disques sur une autre aiguille, en ne déplaçant qu'un seul disque à la fois,

Received by the editors in July 2014 .

Communicated by J. Doyen.

2010 Mathematics Subject Classification : 05C12.

Key words and phrases : Tours de Hanoï, Conjecture de Frame-Stewart. 
d'une aiguille vers une autre, et sans jamais poser un disque sur un disque plus petit. Dudeney précise également que ceci doit être effectué en un nombre minimum de mouvements, et demande quel est ce nombre.

Dudeney montre (au moins pour certaines valeurs de $N$ ) qu'on peut transférer $N$ disques en

$$
\Phi(N)=2^{\nabla 0}+2^{\nabla 1}+\cdots+2^{\nabla(N-1)}
$$

mouvements, où $\nabla n$ désigne la "racine triangulaire" (par défaut) de $n$, c'est-àdire le plus grand entier $p$ tel que $p(p+1) / 2 \leqslant n$.

L'argument de Dudeney a été repris et précisé par Frame et Stewart en 1941 [Fra, St1, St2] et étendu à un nombre quelconque d'aiguilles $\geqslant 3$. Je rappelle ici en quoi consiste cet argument. D'abord, des calculs simples montrent que $\Phi$ vérifie l'équation fonctionnelle

$$
\Phi(N)=\operatorname{Min}_{0 \leqslant M<N} 2 \Phi(M)+2^{N-M}-1 \quad(N \geqslant 1)
$$

ce qui fournit l'algorithme récursif suivant, pour transférer $N$ disques d'une colonne vers une autre, en $\Phi(N)$ mouvements.

Pour déplacer $N$ disques (avec $N>0$ ) de la colonne $a$ vers la colonne $b$, on suppose le problème résolu quand le nombre de disques est strictement plus petit, et on choisit un entier $M<N$ qui réalise le minimum dans la formule ci-dessus. En premier lieu, on déplace les $M$ plus petits disques de $a$ vers une colonne intermédiaire $c$, différente de $a$ et $b$, ce qui demande $\Phi(M)$ mouvements; puis on déplace les $N-M$ disques restants de $a$ vers $b$ sans utiliser la colonne $c$, ce qui demande $2^{N-M}-1$ mouvements (puisqu'il n'y a plus que trois aiguilles disponibles); enfin, on transfère à nouveau les $M$ plus petits disques, cette fois de $c$ vers $b$, ce qui demande encore $\Phi(M)$ mouvements. Ainsi, les $N$ disques ont été transférés de $a$ vers $b$ en

$$
2 \Phi(M)+2^{N-M}-1=\Phi(N)
$$

mouvements, à cause de notre choix de $M$.

Ce raisonnement montre qu'on peut transférer $N$ disques d'une colonne vers une autre en $\Phi(N)$ mouvements, mais ne permet nullement d'affirmer que ce nombre est optimal. Ce point crucial a immédiatement été noté par O. Dunkel [Dun] à propos des "solutions" de Frame et Stewart.

Le but du présent article est de démontrer que, dans le problème de Dudeney, c'est-à-dire avec quatre aiguilles, toute façon de transférer $N$ disques d'une aiguille vers une autre demande au moins $\Phi(N)$ mouvements. Les solutions récursives proposées par Dudeney et Frame-Stewart pour quatre aiguilles sont donc bien optimales. Il faut dire que c'était considéré comme très vraisemblable, essentiellement à cause des calculs sur ordinateur, qui ont vérifié la conjecture jusqu'à $N=30$ [BH, KF]. Il y avait aussi, en faveur de la conjecture, les arguments non rigoureux, mais très plausibles, donnés par Frame et Stewart (discutés dans [H+], $\S 5.2)$ et, dans une moindre mesure, des minorations asymptotiques du nombre de mouvements qui sont assez proches de $\Phi(N)$ quand $N$ est grand; voir [Sze, CS]. Le lecteur désireux d'en savoir plus sur le problème de Dudeney et ses variantes pourra se reporter à l'article de Stockmeyer [Sto], ou au Chapitre 5 du livre [H+]. 


\section{Définitions et résultats préliminaires}

On note $\mathbb{N}$ l'ensemble des entiers naturels $\{0,1,2, \ldots\}$, comme d'habitude. Pour tout entier naturel $n$, on note $[n]$ l'ensemble des $n$ plus petits entiers naturels, $c^{\prime}$ est-à-dire $\{0,1, \ldots, n-1\}$. En particulier $[0]=\varnothing$. Pour $z$ nombre réel, on note $z^{+}$sa partie positive, c'est-à-dire $\max (z, 0)$.

On définit $\Delta n=1+2+\cdots+n=n(n+1) / 2$, pour $n \in \mathbb{N}$; les nombres de cette forme sont les nombres triangulaires: $0,1,3,6,10,15,21 \ldots$ Ils jouent un rôle particulier dans le problème de Dudeney. Pour $n \in \mathbb{N}$, on note $\nabla n$ la racine triangulaire (par défaut) de $n$, c'est-à-dire le plus grand $p \in \mathbb{N}$ tel que $\Delta p \leqslant n$. On a par exemple $\nabla 8=3$, parce que $\Delta 3 \leqslant 8<\Delta 4$. Les fonctions $\Delta: \mathbb{N} \rightarrow \mathbb{N}$ et $\nabla: \mathbb{N} \rightarrow \mathbb{N}$ sont en correspondance galoisienne:

$$
\forall m, n \in \mathbb{N} \quad \Delta m \leqslant n \Longleftrightarrow m \leqslant \nabla n .
$$

Voici leurs premières valeurs:

\begin{tabular}{c|ccccccccccc}
$n$ & 0 & 1 & 2 & 3 & 4 & 5 & 6 & 7 & 8 & 9 & 10 \\
\hline$\Delta n$ & 0 & 1 & 3 & 6 & 10 & 15 & 21 & 28 & 36 & 45 & 55 \\
\hline$\nabla n$ & 0 & 1 & 1 & 2 & 2 & 2 & 3 & 3 & 3 & 3 & 4
\end{tabular}

La fonction $\Phi: \mathbb{N} \rightarrow \mathbb{N}$ est définie par la formule (1.1). En voici les premières valeurs:

$$
\begin{array}{c|ccccccccccc}
n & 0 & 1 & 2 & 3 & 4 & 5 & 6 & 7 & 8 & 9 & 10 \\
\hline \Phi(n) & 0 & 1 & 3 & 5 & 9 & 13 & 17 & 25 & 33 & 41 & 49
\end{array}
$$

Lemme 2.1. Si $n, p$ sont deux entiers naturels tels que $p \leqslant n+1$, on a

$$
\Phi(\Delta n+p)=1+(n+p-1) 2^{n} .
$$

Cette formule est bien connue, et se déduit sans peine de (1.1). Par exemple, en prenant $n=10$ et $p=9$, on obtient $\Phi(64)=1+18 \cdot 2^{10}=18433$.

L'identité (1.2) est également classique, mais moins facile à vérifier. Dans le présent article, j'en utiliserai une forme affaiblie, à savoir, l'inégalité

$$
\forall a, b \in \mathbb{N} \quad \Phi(a+b) \leqslant 2 \Phi(a)+2^{b}-1 .
$$

On définit maintenant une fonction $\Psi: \mathcal{P}_{\text {fin }}(\mathbb{N}) \rightarrow \mathbb{N}$, où $\mathcal{P}_{\text {fin }}(\mathbb{N})$ désigne le treillis des parties finies de $\mathbb{N}$, qui jouera un rôle essentiel.

Soit $E$ une partie finie de $\mathbb{N}$. Pour tout entier naturel $L$, posons

$$
\Psi_{L}(E)=(1-L) 2^{L}-1+\sum_{n \in E} 2^{\min (\nabla n, L)}
$$

et définissons

$$
\Psi(E)=\operatorname{Sup}_{L \in \mathbb{N}} \Psi_{L}(E) .
$$

Comme $\Psi_{L}(E) \rightarrow-\infty$ quand $L \rightarrow \infty$, cette borne supérieure est en fait un maximum, et $\Psi(E)$ est un entier; un entier relatif, a priori, mais il est minoré par $\Psi(\varnothing)=0$, les fonctions $\Psi_{L}$ et $\Psi$ étant évidemment croissantes pour l'inclusion. 
Lemme 2.2. Pour tout entier naturel $n$,

$$
\Psi[n]=\frac{\Phi(n+1)-1}{2}=\frac{1}{2}\left(2^{\nabla 1}+2^{\nabla 2}+\cdots+2^{\nabla n}\right) .
$$

Démonstration. On a déjà vu que $\Psi[0]=0$. Supposons donc $n \geqslant 1$, et écrivons $n=\Delta m+p$ avec $m=\nabla n$, si bien que $m \geqslant 1$ et $0 \leqslant p \leqslant m$. Par le lemme 2.1,

$$
\begin{aligned}
\Phi(\Delta m) & =1+(m-1) 2^{m} \\
\Phi(n+1) & =1+(m+p) 2^{m}
\end{aligned}
$$

Pour tout entier naturel $L$, on a

$$
\begin{aligned}
\Psi_{L+1}[n]-\Psi_{L}[n] & =-(L+1) 2^{L}+\sum_{k \in[n]} 2^{\min (\nabla k, L+1)}-2^{\min (\nabla k, L)} \\
& =2^{L}[\#\{k \in[n]: k \geqslant \Delta(L+1)\}-(L+1)] \\
& =2^{L}\left[[n-\Delta(L+1)]^{+}-(L+1)\right]
\end{aligned}
$$

Cette expression est strictement positive si et seulement si

$$
n \geqslant \Delta(L+1)+L+2=\Delta(L+2)
$$

ce qui équivaut à $\nabla n \geqslant L+2$, ou encore $L<m-1$. Par conséquent, la suite des $\Psi_{L}[n]$ atteint son maximum pour $L=m-1$, et

$$
\begin{aligned}
\Psi[n] & =\Psi_{m-1}[n]=(2-m) 2^{m-1}-1+\sum_{0 \leqslant k<\Delta m} 2^{\nabla k}+\sum_{\Delta m \leqslant k<n} 2^{m-1} \\
& =(2-m) 2^{m-1}-1+\Phi(\Delta m)+(n-\Delta m) 2^{m-1} \\
& =(2-m) 2^{m-1}+(m-1) 2^{m}+p 2^{m-1} \\
& =(m+p) 2^{m-1}=\frac{\Phi(n+1)-1}{2}
\end{aligned}
$$

ce qu'il fallait démontrer.

De ce résultat et de l'inégalité classique (2.1) pour $\Phi$, on déduit une inégalité fonctionnelle similaire pour $\Psi$ :

$$
\forall a, b \in \mathbb{N} \quad \Psi[a+b] \leqslant 2 \Psi[a]+2^{b-1} .
$$

Lemme 2.3. On a $\Psi[n+2] \geqslant 2^{(\nabla n)+1}$ pour tout $n$ entier naturel.

Démonstration. Soit $s=\nabla n$. Comme $\Psi[\cdot]$ est croissante, il suffit de montrer que $\Psi[\Delta s+2] \geqslant 2^{s+1}$. Cette inégalité est satisfaite (avec égalité) pour $s=0$ et 1 , car $\Psi[2]=2$ et $\Psi[3]=4$. Pour $s \geqslant 2$, les Lemmes 2.2 et 2.1 donnent $\Psi[\Delta s+2]=$ $(s+2) 2^{s-1}$, qui est $\geqslant 2^{s+1}$ puisque $s+2 \geqslant 4$. 
Lemme 2.4. Pour toute partie finie $E$ de $\mathbb{N}$, on a

$$
n \leqslant \Psi[n] \leqslant \Psi(E) \leqslant 2^{n}-1
$$

où n est le cardinal de E.

Démonstration. Première inégalité. On a $\Psi[n] \geqslant \Psi_{0}[n]=n$.

Deuxième inégalité. Soient $e_{0}<e_{1}<\cdots<e_{n-1}$ les $n$ éléments de $E$. Comme $e_{k} \geqslant k$ pour tout $k$, on a

$$
\begin{aligned}
\Psi_{L}(E) & =(1-L) 2^{L}-1+\sum_{0 \leqslant k<n} 2^{\min \left(\nabla e_{k}, L\right)} \\
& \geqslant(1-L) 2^{L}-1+\sum_{0 \leqslant k<n} 2^{\min (\nabla k, L)}=\Psi_{L}[n]
\end{aligned}
$$

pour tout $L$, et donc $\Psi(E) \geqslant \Psi[n]$.

Troisième inégalité. Pour tout $L$ entier naturel,

$$
\begin{aligned}
\Psi_{L}(E) & =(1-L) 2^{L}-1+\sum_{k \in E} 2^{\min (\nabla k, L)} \\
& \leqslant(1-L) 2^{L}-1+\sum_{k \in E} 2^{L}=(1+n-L) 2^{L}-1
\end{aligned}
$$

Mais on sait que $2^{s} \geqslant 1+s$ pour tout entier relatif $s$, ce qui donne $2^{n} \geqslant(1+n-L) 2^{L}$ en prenant $s=n-L$, et par conséquent $\Psi_{L}(E) \leqslant 2^{n}-1$.

Lemme 2.5. Soient $A, B$ deux parties finies de $\mathbb{N}$. On a

$$
\Psi(A)-\Psi(B) \leqslant \sum_{k \in A-B} 2^{\nabla k}
$$

Démonstration. Soit $L$ un entier naturel pour lequel $\Psi(A)=\Psi_{L}(A)$. Alors,

$$
\begin{aligned}
\Psi(A)-\Psi(B) & \leqslant \Psi_{L}(A)-\Psi_{L}(B) \leqslant \Psi_{L}(A)-\Psi_{L}(A \cap B) \\
& =\sum_{k \in A-B} 2^{\min (\nabla k, L)} \leqslant \sum_{k \in A-B} 2^{\nabla k}
\end{aligned}
$$

ce qu'il fallait démontrer.

Lemme 2.6. Soit $A$ une partie finie de $\mathbb{N}$, et s un entier naturel tel que l'ensemble $A-[\Delta s]$ contienne au plus s éléments. Alors

$$
\Psi(A)-\Psi(A-\{a\}) \leqslant 2^{s-1}
$$

pour tout a dans $A$. 
Démonstration. On peut supposer $A$ non vide, et donc $s \geqslant 1$. Alors, pour tout $L \geqslant s-1$,

$$
\begin{aligned}
\Psi_{L+1}(A)-\Psi_{L}(A) & =2^{L}[\#\{n \in A: n \geqslant \Delta(L+1)\}-(L+1)] \\
& \leqslant 2^{L}[\#\{n \in A: n \geqslant \Delta s\}-s] \leqslant 0
\end{aligned}
$$

par hypothèse, ce qui montre que la suite des $\Psi_{L}(A)$ est décroissante à partir de $L=s-1$. Il existe donc un $L \leqslant s-1$ tel que $\Psi(A)=\Psi_{L}(A)$, et

$$
\Psi(A)-\Psi(A-\{a\}) \leqslant \Psi_{L}(A)-\Psi_{L}(A-\{a\})=2^{\min (\nabla a, L)} \leqslant 2^{L} \leqslant 2^{s-1}
$$

ce qu'il fallait démontrer.

Lemme 2.7. Soient $n, s$ deux entiers naturels tels que $s \geqslant 1$ et $n \geqslant \Delta(s-1)$, et $A$ une partie de $[n]$. Alors

$$
\Psi\left(A \cup\left\{b_{1}, \ldots, b_{s}\right\}\right)-\Psi(A) \leqslant \Psi[n+s]-\Psi[n]
$$

quels que soient $b_{1}, \ldots, b_{s}$ (non nécessairement distincts) dans $\mathbb{N}$.

Démonstration. Posons $A_{t}=A \cup\left\{b_{1}, \ldots, b_{t}\right\}$ pour $0 \leqslant t \leqslant s$. Il suffit de montrer que

$$
\Psi\left(A_{t}\right)-\Psi\left(A_{t-1}\right) \leqslant \Psi[n+t]-\Psi[n+t-1]
$$

pour tout $t$ tel que $1 \leqslant t \leqslant s$. Bien sûr, on peut supposer que l'inclusion de $A_{t-1}$ dans $A_{t}$ est stricte. Par le Lemme 2.2, le membre de droite s'écrit $2^{\sigma-1}$, où $\sigma=\nabla(n+t)$, et l'inégalité résultera du Lemme 2.6, à condition de montrer que l'ensemble $A_{t}-[\Delta \sigma]$ est de cardinal $\leqslant \sigma$. Notons d'abord que $\Delta(\sigma+1)>n+t$, ce qui revient à dire que $\Delta \sigma+\sigma \geqslant n+t$. Ensuite,

$$
\begin{aligned}
\#\left(A_{t}-[\Delta \sigma]\right) & \leqslant t+\#([n]-[\Delta \sigma])=t+(n-\Delta \sigma)^{+} \\
& =\max (t, t+n-\Delta \sigma) \leqslant \max (t, \sigma)
\end{aligned}
$$

et il reste à vérifier que $t \leqslant \sigma$. Pour cela, observons que

$$
\Delta t-t=\Delta(t-1) \leqslant \Delta(s-1) \leqslant n
$$

et donc $\Delta t \leqslant n+t$, ce qu'il fallait démontrer.

Lemme 2.8. Soient $A, B$ deux parties finies de $\mathbb{N}$. On a

$$
\begin{aligned}
\Psi(A)+\Psi(B) & \geqslant \frac{\Phi(n+3)-5}{4}=\frac{1}{2} \Psi[n+2]-1 \\
& =\frac{1}{4}\left(2^{\nabla 3}+2^{\nabla 4}+\cdots+2^{\nabla(n+2)}\right)
\end{aligned}
$$

où n est le cardinal de $A \cup B$. 
Démonstration. Notons $E=A \cup B$. Soit $L$ un entier naturel quelconque. On a vu, dans la preuve du Lemme 2.4, qu'on a toujours $\Psi_{L}(E) \geqslant \Psi_{L}[n]$. Par conséquent,

$$
\begin{aligned}
\Psi(A)+\Psi(B) & \geqslant \Psi_{L}(A)+\Psi_{L}(B)=\Psi_{L}(A \cap B)+\Psi_{L}(A \cup B) \\
& \geqslant \Psi_{L}(\varnothing)+\Psi_{L}(E) \geqslant \Psi_{L}[0]+\Psi_{L}[n] .
\end{aligned}
$$

Ecrivons maintenant $n+3=\Delta m+p$, avec $m=\nabla(n+3)$, si bien que $m \geqslant 2$ et $0 \leqslant p \leqslant m$. On voit que $n \geqslant \Delta(m-2)$, et

$$
\begin{aligned}
\Phi(n+3) & =1+(m+p-1) 2^{m} \\
\Phi(\Delta(m-2)) & =1+(m-3) 2^{m-2}
\end{aligned}
$$

Prenons maintenant $L=m-2$. On a

$$
\begin{aligned}
\Psi_{L}[0]+\Psi_{L}[n] & =(1-L) 2^{L+1}-2+\sum_{0 \leqslant k<n} 2^{\min (\nabla k, L)} \\
& =(3-m) 2^{m-1}-2+\sum_{0 \leqslant k<\Delta(m-2)} 2^{\nabla k}+\sum_{\Delta(m-2) \leqslant k<n} 2^{m-2} \\
& =(3-m) 2^{m-1}-2+\Phi(\Delta(m-2))+(n-\Delta(m-2)) 2^{m-2} \\
& =(3-m) 2^{m-1}-1+(m-3) 2^{m-2}+(p+2 m-4) 2^{m-2} \\
& =(m+p-1) 2^{m-2}-1=\frac{\Phi(n+3)-5}{4}
\end{aligned}
$$

d'où le résultat.

Revenons au problème de Dudeney. L'ensemble des tiges (ou des colonnes), noté $\mathcal{C}$, sera un quelconque ensemble à quatre éléments, par exemple $\{0,1,2,3\}$. Les $N$ disques seront représentés par les entiers de 0 à $N-1$, les plus grands entiers correspondant aux plus grands disques.

Un empilement (de $N$ disques sur $\mathcal{~ C ) ~ d e ́ s i g n e r a ~ u n e ~ c o n f i g u r a t i o n ~ a u t o r i s e ́ e ~ d u ~ j e u , ~}$ c'est-à-dire une manière de répartir les $N$ disques entre les quatre colonnes, où dans chaque colonne les disques sont empilés par taille décroissante. Un empilement peut être représenté par un élément (unique, et arbitraire) de $\mathrm{C}^{[N]}$, c'est-àdire une fonction

$$
[N] \longrightarrow \mathcal{C}
$$

quelconque (il y en a donc $4^{N}$ en tout). Cette fonction indique dans quelle colonne se trouve chacun des disques.

La distance $d(\mathbf{u}, \mathbf{v})$ entre deux empilements $\mathbf{u}, \mathbf{v}:[N] \rightarrow \mathcal{C}$ est le nombre minimum de mouvements de disques pour passer de $\mathbf{u}$ à $\mathbf{v}$, en respectant les règles du jeu bien sûr. Le résultat principal de cet article est le suivant.

Théorème 2.9. On pose $\mathcal{C}=\{0,1,2,3\}$. Soit $N$ un entier naturel, et $\mathbf{u}, \mathbf{v}:[N] \rightarrow \mathcal{C}$ deux empilements de $N$ disques. On suppose que dans l'empilement $\mathbf{v}$, les colonnes 0 et 1 sont vides, c'est-à-dire $\mathbf{v}[N] \subseteq\{2,3\}$. Alors

$$
d(\mathbf{u}, \mathbf{v}) \geqslant \Psi\{k \in[N]: \mathbf{u}(k)=0\} .
$$

La démonstration de ce théorème constitue le chapitre suivant de cet article. 


\section{Démonstration du théorème}

\subsection{Préliminaires}

On fixe le nombre de disques $N$, et on suppose que le résultat est vrai pour tout nombre de disques strictement plus petit. Soient $\mathbf{u}, \mathbf{v}$ deux empilements de $N$ disques, avec $\mathbf{v}[N] \subseteq\{2,3\}$. On note $E=\{k \in[N]: \mathbf{u}(k)=0\}$, c'est-à-dire le contenu de la colonne 0 dans l'empilement $\mathbf{u}$. On peut supposer $E$ non vide, sinon c'est trivial, et en particulier $N \geqslant 1$.

Si $\mathbf{u}^{\prime}, \mathbf{v}^{\prime}:[N-1] \rightarrow \mathcal{C}$ désignent les restrictions à $[N-1]$ des fonctions $\mathbf{u}, \mathbf{v}$ (on "oublie" le plus grand disque), ces empilements n'ont que $N-1$ disques, et on peut leur appliquer l'hypothèse de récurrence:

$$
\begin{aligned}
d(\mathbf{u}, \mathbf{v}) \geqslant d\left(\mathbf{u}^{\prime}, \mathbf{v}^{\prime}\right) & \geqslant \Psi\left\{k \in[N-1]: \mathbf{u}^{\prime}(k)=0\right\} \\
& =\Psi\{k \in[N-1]: \mathbf{u}(k)=0\} \\
& =\Psi(E-\{N-1\}) .
\end{aligned}
$$

Si $E$ ne contient pas $N-1$, il vient $d(\mathbf{u}, \mathbf{v}) \geqslant \Psi E$, qui est le résultat cherché. Ce cas trivial étant réglé, je supposerai dans toute la suite que $E$ contient $N-1$, c'est-àdire que le plus grand disque de l'empilement $\mathbf{u}$ est dans la colonne 0 . Ce même disque, dans l'empilement $\mathbf{v}$, se trouvera dans la colonne 2 ou 3 . Je peux supposer sans perte de généralité que c'est la colonne 2 , i.e. $\mathbf{u}(N-1)=0$ et $\mathbf{v}(N-1)=2$. Notons $D$ la distance entre $\mathbf{u}$ et $\mathbf{v}$, et soit $\gamma:[D+1] \rightarrow \mathcal{C}^{[N]}$ un chemin géodésique allant de $\mathbf{u}$ à $\mathbf{v}$ dans le graphe $\mathcal{e}^{[N]}$, c'est-à-dire que $\gamma(0)=\mathbf{u}, \gamma(D)=\mathbf{v}$, et $d(\gamma(i), \gamma(j))=|i-j|$ pour tous $i, j$ dans $[D+1]$. Par la suite, je noterai parfois $\gamma_{i}$ au lieu de $\gamma(i)$.

Notons $E^{\prime}=\left\{k \in E: \exists t \in[D+1] \gamma_{t}(k)=3\right\}$, c'est-à-dire l'ensemble des disques qui sont initialement dans la colonne 0 , et qui passent au moins une fois dans la colonne 3.

Considérons d'abord le cas où $E^{\prime}$ est vide. Cela signifie que les disques de $E$ ne passent jamais dans la colonne 3. En particulier, ils finissent leur course dans la colonne 2. Et comme ils ne peuvent utiliser que trois tiges parmi les quatre, leur déplacement demande au moins $2^{\# E}-1$ mouvements, ce qui implique $D \geqslant$ $2^{\# E}-1$. Mais on sait d'autre part que $\Psi E \leqslant 2^{\# E}-1$, par le Lemme 2.4, donc l'inégalité $D \geqslant \Psi E$ est clairement satisfaite dans ce cas.

On supposera donc désormais que $E^{\prime}$ est non vide, et on notera $T$ son plus grand élément. Définissons également l'ensemble $E^{\prime \prime}=\{k \in E: k>T\}$; on notera $K$ son cardinal (qui peut être nul), et $b_{1}<b_{2}<\cdots<b_{K}$ ses éléments. On voit que

$$
E \subseteq \underbrace{[T] \sqcup\{T\}}_{\text {contient } E^{\prime}} \sqcup \underbrace{\left\{b_{1}, \ldots, b_{K}\right\}}_{E^{\prime \prime}} \subseteq[N]
$$

et en particulier

$$
T+K+1 \leqslant N
$$

Soit $t_{0}$ le premier instant où le disque $T$ n'est plus dans la colonne $0, c^{\prime}$ est-à-dire le plus petit élément de $[D+1]$ pour lequel $\gamma_{t_{0}}(T) \neq 0$. Comme c'est le plus petit, 
on a $\gamma_{t_{0}-1}(T)=0$, ce qui veut dire qu'entre les dates $t_{0}-1$ et $t_{0}$, c'est le disque $T$ qui est déplacé, de la colonne 0 vers une autre. Mais un tel mouvement n'est possible que si la colonne 0 et la colonne destination ne contiennent aucun disque $<T$. Notons $\mathbf{x}_{0}=\gamma\left(t_{0}-1\right)$, c'est-à-dire l'empilement juste avant le mouvement du disque $T$. Dans cet empilement, la colonne 0 contient le disque $T$ et aucun disque $<T$, et une autre colonne ne contient aucun disque $\leqslant T$.

Soit $t_{1}$ le premier instant où le disque $T$ se trouve dans la colonne 3 , c'est-à-dire le plus petit entier pour lequel $\gamma_{t_{1}}(T)=3$, et $\mathbf{x}_{3}=\gamma\left(t_{1}\right)$ l'empilement correspondant. Par des arguments similaires, dans cet empilement la colonne 3 contient le disque $T$ et aucun disque $<T$, et une autre colonne ne contient aucun disque $\leqslant T$. Les dates $t_{0}, t_{1}$ satisfont $1 \leqslant t_{0} \leqslant t_{1} \leqslant D$.

Soit $t_{2}$ le premier instant où le disque $N-1$ n'est plus dans la colonne 0 , i.e. $\gamma_{t_{2}}(N-1) \neq 0$, et $\mathbf{z}_{0}=\gamma\left(t_{2}-1\right)$. Dans cet empilement, la colonne 0 contient uniquement le disque $N-1$, et une autre colonne est vide. Enfin, soit $t_{3}$ le dernier instant où le disque $N-1$ n'est pas dans la colonne 2, i.e. $\gamma_{t_{3}}(N-1) \neq 2$, et $\mathbf{z}_{2}=\gamma\left(t_{3}+1\right)$. Dans cet empilement, la colonne 2 contient uniquement le disque $N-1$, et une autre colonne est vide. Les dates $t_{2}, t_{3}$ satisfont $1 \leqslant t_{2} \leqslant t_{3}+1 \leqslant D$.

Comme pour $\mathbf{u}$ et $\mathbf{v}$, les notations $\mathbf{x}_{a}^{\prime}, \mathbf{z}_{b}^{\prime}$ désigneront les restrictions des $\mathbf{x}_{a}, \mathbf{z}_{b}$ à $[N-1]$, c'est-à-dire en oubliant le plus grand disque. Je noterai d'autre part $\mathbf{u}^{\prime \prime}, \mathbf{v}^{\prime \prime}, \mathbf{x}_{a}^{\prime \prime}, \mathbf{z}_{b}^{\prime \prime}$ les empilements restreints à $[T], c^{\prime}$ est-à-dire en oubliant tous les disques $\geqslant T$.

On observe que dans l'empilement $\mathbf{z}_{0}^{\prime}$, la colonne 0 est vide ainsi qu'une autre, ce qui permet d'appliquer l'hypothèse de récurrence:

$$
\begin{aligned}
d\left(\mathbf{u}, \mathbf{z}_{0}\right) \geqslant d\left(\mathbf{u}^{\prime}, \mathbf{z}_{0}^{\prime}\right) & \geqslant \Psi\{k \in[N-1]: \mathbf{u}(k)=0\} \\
& =\Psi(E-\{N-1\})
\end{aligned}
$$

et de manière similaire,

$$
d\left(\mathbf{u}, \mathbf{x}_{0}\right) \geqslant d\left(\mathbf{u}^{\prime \prime}, \mathbf{x}_{0}^{\prime \prime}\right) \geqslant \Psi(E \cap[T]) .
$$

D'ailleurs, quand $T=N-1$ c'est exactement la même inégalité.

\subsection{Le cas $\Delta K>T$}

Examinons d'abord le cas $\Delta K>T$, qui est le plus simple. Tout d'abord, cela entraîne $K \geqslant 1$, et $T<b_{K}=N-1$; le plus grand disque ne passe jamais par la colonne 3. Ensuite, l'ensemble $\{x \in E: x \geqslant \Delta K\}$ est contenu dans $\{x \in E$ : $x>T\}=E^{\prime \prime}$ et donc de cardinal au plus $K$, ce qui entraîne

$$
\Psi E-\Psi(E-\{N-1\}) \leqslant 2^{K-1}
$$

par le Lemme 2.6. Avec (3.3), il en résulte que

$$
d\left(\mathbf{u}, \mathbf{z}_{0}\right) \geqslant \Psi E-2^{K-1} .
$$


Comme $t_{2} \leqslant t_{3}+1$, le chemin $\gamma$ parcourt les configurations $\mathbf{u}, \mathbf{z}_{0}, \mathbf{z}_{2}, \mathbf{v}$ dans l'ordre suivant (au sens large, certains empilements peuvent coïncider):

$$
\mathbf{u} \longrightarrow \mathbf{z}_{0} \longrightarrow \mathbf{z}_{2} \longrightarrow \mathbf{v}
$$

Dans l'empilement $\mathbf{z}_{2}$, la colonne 2 et la colonne $c=\gamma_{t_{3}}(N-1)$, qui vaut 0 ou 1 , ne contiennent aucun disque $<N-1$. Les disques $b_{1}, \ldots, b_{K-1}$ sont donc tous dans la colonne $1-c$. Dans l'empilement final $\mathbf{v}$, tous ces disques devront être dans la colonne 2. Le déplacement de ces disques, qui n'ont pas le droit de passer dans la colonne 3 , demandera au moins $2^{K-1}-1$ mouvements:

$$
d\left(\mathbf{z}_{2}, \mathbf{v}\right) \geqslant 2^{K-1}-1
$$

Enfin $d\left(\mathbf{z}_{0}, \mathbf{z}_{2}\right) \geqslant 1$, car le disque $N-1$ doit passer de la colonne 0 à la colonne 2, et donc

$$
\begin{aligned}
D=d(\mathbf{u}, \mathbf{v}) & =d\left(\mathbf{u}, \mathbf{z}_{0}\right)+d\left(\mathbf{z}_{0}, \mathbf{z}_{2}\right)+d\left(\mathbf{z}_{2}, \mathbf{v}\right) \\
& \geqslant\left(\Psi E-2^{K-1}\right)+1+\left(2^{K-1}-1\right)=\Psi E
\end{aligned}
$$

ce qu'il fallait démontrer.

\subsection{Le cas $\Delta K \leqslant T$}

Nous devons à présent traiter le cas $\Delta K \leqslant T$.

Maintenant l'inégalité (3.5) n'est plus valable, mais on a une inégalité similaire

$$
\Psi E-\Psi(E-\{N-1\}) \leqslant 2^{\nabla(T+K+1)-1}
$$

qui peut elle aussi se déduire du Lemme 2.6, en montrant que $E-[\Delta s]$ contient au plus $s$ éléments, où $s=\nabla(T+K+1)$. Comme $E$ est inclus dans $[T+1] \cup E^{\prime \prime}$, la différence $E-[\Delta s]$ contient au plus $(T+1-\Delta s)^{+}+K$ éléments, et il suffit de montrer que ce nombre est $\leqslant s$. Cela revient à dire qu'on a les deux inégalités

$$
\left\{\begin{array}{r}
K \leqslant s \\
T+1-\Delta s+K \leqslant s
\end{array}\right.
$$

La deuxième inégalité équivaut à $T+K+1<\Delta(s+1)$, une condition automatiquement satisfaite vu la définition de $s$. Quant à la première inégalité, elle résulte de ce que $s \geqslant \nabla T \geqslant K$. Ceci termine la démonstration de la formule (3.7).

Avec (3.3), il en résulte que

$$
d\left(\mathbf{u}, \mathbf{z}_{0}\right) \geqslant d\left(\mathbf{u}^{\prime}, \mathbf{z}_{0}^{\prime}\right) \geqslant \Psi E-2^{\nabla(T+K+1)-1} .
$$

On peut avoir $K=0$, ce qui équivaut à $T=N-1$. Nous allons examiner en premier ce cas particulier. Cela signifie que le plus grand disque, qui est initialement dans la colonne 0 , et finalement dans la colonne 2, passe au moins une fois dans 
la colonne 3. Ici $T$ et $N-1$ sont le même disque, donc $t_{1} \leqslant t_{3}$, et le chemin $\gamma$ parcourt les configurations dans l'ordre suivant:

$$
\mathbf{u} \longrightarrow \mathbf{z}_{0}=\mathbf{x}_{0} \longrightarrow \mathbf{x}_{3} \longrightarrow \mathbf{z}_{2} \longrightarrow \mathbf{v}
$$

Notons $c=\gamma_{t_{3}}(N-1)$, qui est $\neq 2$. Dans l'empilement $\mathbf{z}_{2}^{\prime}$, les colonnes 2 et $c$ sont vides, et tous les disques sont dans les deux autres colonnes. Ecrivons

$$
\{0,1,2,3\}-\{2, c\}=\{a, b\}
$$

avec $a \in\{0,3\}$ et $b \in\{0,1\}$, selon la table suivante:

$$
\begin{array}{c|ccc}
c & 0 & 1 & 3 \\
\hline a & 3 & 3 & 0 \\
\hline b & 1 & 0 & 1
\end{array}
$$

Définissons maintenant

$$
\begin{aligned}
A & =\left\{k \in[N-1]: \mathbf{z}_{2}(k)=a\right\} \\
B & =\left\{k \in[N-1]: \mathbf{z}_{2}(k)=b\right\} .
\end{aligned}
$$

Ces ensembles vérifient $A \sqcup B=[N-1]$ donc, par le Lemme 2.8,

$$
\begin{aligned}
\Psi A+\Psi B & \geqslant \frac{1}{2} \Psi[N+1]-1 \\
& =\frac{1}{4}\left(2^{\nabla(N+1)}+2^{\nabla N}\right)+\frac{1}{2} \Psi[N-1]-1 \\
& \geqslant 2^{\nabla(T+K+1)-1}+\frac{1}{2} \Psi[N-1]-1 .
\end{aligned}
$$

Dans l'empilement $\mathbf{x}_{a}^{\prime}$, la colonne $a$ est vide ainsi qu'une autre, ce qui permet d'appliquer l'hypothèse de récurrence

$$
d\left(\mathbf{z}_{2}^{\prime}, \mathbf{x}_{a}^{\prime}\right) \geqslant \Psi A
$$

et de même, dans $\mathbf{v}^{\prime}$ la colonne $b$ est vide ainsi que la colonne $1-b$, donc

$$
d\left(\mathbf{z}_{2}, \mathbf{v}\right) \geqslant d\left(\mathbf{z}_{2}^{\prime}, \mathbf{v}^{\prime}\right) \geqslant \Psi B
$$

Entre les empilements $\mathbf{z}_{0}$ et $\mathbf{z}_{2}$, les disques $<N-1$ effectuent au moins $d\left(\mathbf{x}_{a}^{\prime}, \mathbf{z}_{2}^{\prime}\right)$ mouvements, et le disque $N-1$ effectue au moins deux mouvements (pour passer dans les colonnes $0,3,2$ ), donc

$$
d\left(\mathbf{z}_{0}, \mathbf{z}_{2}\right) \geqslant \Psi A+2
$$

et finalement

$$
\begin{aligned}
D=d(\mathbf{u}, \mathbf{v}) & =d\left(\mathbf{u}, \mathbf{z}_{0}\right)+d\left(\mathbf{z}_{0}, \mathbf{z}_{2}\right)+d\left(\mathbf{z}_{2}, \mathbf{v}\right) \\
& \geqslant \Psi E-2^{\nabla(T+K+1)-1}+\Psi A+2+\Psi B \\
& \geqslant \Psi E+1+\frac{1}{2} \Psi[N-1] \geqslant \Psi E
\end{aligned}
$$

ce qu'il fallait démontrer. 
On supposera donc maintenant $K \geqslant 1$ (et toujours $\Delta K \leqslant T$ ), d'où $T<b_{K}=N-1$ (le plus grand disque ne passe jamais par la colonne 3 ).

Maintenant, rien ne permet de comparer $t_{1}$ et $t_{3}+1$, les dates où apparaissent les empilements $\mathbf{x}_{3}$ et $\mathbf{z}_{2}$ respectivement. On peut seulement dire que ces deux instants sont différents, car le dernier disque déplacé n'est pas le même dans les deux cas. Cela nous oblige à une dernière disjonction de cas, selon la position relative de ces deux instants.

Supposons d'abord $t_{1}>t_{3}+1$.

Le chemin $\gamma$ parcourt les configurations dans l'ordre suivant:

$$
\mathbf{u} \longrightarrow \mathbf{z}_{0} \longrightarrow \mathbf{z}_{2} \longrightarrow \mathbf{x}_{3} \longrightarrow \mathbf{v}
$$

Dans l'empilement $\mathbf{x}_{3}$, la colonne 3 ne contient aucun disque $<T$, et il en est de même pour la colonne $d=\gamma_{t_{1}-1}(T)$. Les disques $<T$ sont donc répartis dans les deux colonnes restantes. Posons $c=\gamma_{t_{3}}(N-1) \in\{0,1\}$ comme précédemment, et écrivons

$$
\{0,1,2,3\}-\{3, d\}=\{a, b\}
$$

avec $a \in\{2, c\}$ et $b \in\{0,1\}$, selon la table suivante:

$$
\begin{array}{c|cc}
d & 0 \text { ou } 1 & 2 \\
\hline a & 2 & c \\
\hline b & 1-d & 1-c
\end{array}
$$

Définissons les ensembles

$$
\begin{aligned}
A & =\left\{k \in[T]: \mathbf{x}_{3}(k)=a\right\} \\
B & =\left\{k \in[T]: \mathbf{x}_{3}(k)=b\right\}
\end{aligned}
$$

qui sont complémentaires dans $[T]$. Dans l'empilement $\mathbf{z}_{2}^{\prime \prime}$, la colonne $a$ est vide ainsi qu'une autre, donc

$$
d\left(\mathbf{x}_{3}, \mathbf{z}_{2}\right) \geqslant d\left(\mathbf{x}_{3}^{\prime \prime}, \mathbf{z}_{2}^{\prime \prime}\right) \geqslant \Psi A
$$

et dans $\mathbf{v}^{\prime \prime}$, la colonne $b$ est vide ainsi que $1-b$, donc

$$
d\left(\mathbf{x}_{3}, \mathbf{v}\right) \geqslant d\left(\mathbf{x}_{3}^{\prime \prime}, \mathbf{v}^{\prime \prime}\right) \geqslant \Psi B
$$

et ici encore $d\left(\mathbf{z}_{0}, \mathbf{z}_{2}\right) \geqslant 1$, donc

$$
\begin{aligned}
D=d(\mathbf{u}, \mathbf{v}) & =d\left(\mathbf{u}, \mathbf{z}_{0}\right)+d\left(\mathbf{z}_{0}, \mathbf{z}_{2}\right)+d\left(\mathbf{z}_{2}, \mathbf{x}_{3}\right)+d\left(\mathbf{x}_{3}, \mathbf{v}\right) \\
& \geqslant \Psi E-2^{\nabla(T+K+1)-1}+1+\Psi A+\Psi B \\
& \geqslant \Psi E-2^{\nabla(T+K+1)-1}+\frac{1}{2} \Psi[T+2]
\end{aligned}
$$

Posons $s=\nabla(T+K+1)$. L'hypothèse $T \geqslant \Delta K$ équivaut à $T+K+1 \geqslant \Delta(K+1)$, soit $s \geqslant K+1$. Mais alors $T=(T+K+1)-(K+1) \geqslant \Delta s-s=\Delta(s-1)$, c'està-dire $\nabla T \geqslant s-1$. Par le Lemme 2.3 , il en résulte que $\Psi[T+2] \geqslant 2^{s}$, et donc $D \geqslant \Psi E$, ce qu'il fallait démontrer. 
Il reste finalement le cas $t_{1}<t_{3}+1$.

Le chemin $\gamma$ parcourt les configurations dans l'ordre suivant:

$$
\mathbf{u} \longrightarrow \mathbf{x}_{0} \longrightarrow \mathbf{x}_{3}, \mathbf{z}_{0} \longrightarrow \mathbf{z}_{2} \longrightarrow \mathbf{v}
$$

Dans l'empilement $\mathbf{z}_{2}^{\prime}$, les colonnes 2 et $c=\gamma_{t_{3}}(N-1) \in\{0,1\}$ sont vides, donc tous les disques sont dans les colonnes 3 et $b=1-c$. En particulier, les disques $b_{1}, \ldots, b_{K-1}$ sont dans la colonne $b$.

Dans l'empilement $\mathbf{x}_{3}^{\prime \prime}$, la colonne 3 est vide ainsi qu'une autre, donc $d\left(\mathbf{z}_{2}^{\prime \prime}, \mathbf{x}_{3}^{\prime \prime}\right) \geqslant$ $\Psi A$, où

$$
A=\left\{k \in[T]: \mathbf{z}_{2}(k)=3\right\}
$$

et dans l'empilement $\mathbf{v}^{\prime}$, la colonne $b$ est vide ainsi que $1-b$, donc

$$
d\left(\mathbf{z}_{2}, \mathbf{v}\right) \geqslant d\left(\mathbf{z}_{2}^{\prime}, \mathbf{v}^{\prime}\right) \geqslant \Psi B
$$

où

$$
\begin{aligned}
B & =\left\{k \in[N-1]: \mathbf{z}_{2}(k)=b\right\} \\
& \supseteq\left\{k \in[T]: \mathbf{z}_{2}(k)=b\right\} \sqcup\left\{b_{1}, \ldots, b_{K-1}\right\} .
\end{aligned}
$$

L'ensemble $A \cup B$ contient $[T] \sqcup\left\{b_{1}, \ldots, b_{K-1}\right\}$, donc possède au moins $T+K-1$ éléments, et

$$
\Psi A+\Psi B \geqslant \frac{1}{2} \Psi[T+K+1]-1 .
$$

Entre $\mathbf{x}_{3}$ et $\mathbf{z}_{2}$, les disques $<T$ effectuent au moins $\Psi A$ mouvements; et, entre $\mathbf{u}$ et $\mathbf{x}_{0}$, ils effectuent au moins $\Psi(E \cap[T])$ mouvements. De plus, dans $\mathbf{u}$ les disques $b_{1}, \ldots, b_{K-1}$ sont tous dans la colonne 0 , tandis que dans $\mathbf{z}_{0}$ ils sont tous dans une autre colonne (qui peut être 1 ou 2). Comme ces disques évitent la colonne 3, ils doivent effectuer au moins $2^{K-1}-1$ mouvements entre $\mathbf{u}$ et $\mathbf{z}_{0}$. Enfin, le disque $T$ effectue au moins un mouvement entre $\mathbf{x}_{0}$ et $\mathbf{x}_{3}$, et le disque $N-1$ effectue au moins un mouvement entre $\mathbf{z}_{0}$ et $\mathbf{z}_{2}$. Le nombre total de mouvements de disques entre $\mathbf{u}$ et $\mathbf{z}_{2}$ satisfait donc la minoration

$$
d\left(\mathbf{u}, \mathbf{z}_{2}\right) \geqslant \Psi(E \cap[T])+\Psi A+2^{K-1}+1
$$

d'où il résulte que

$$
\begin{aligned}
D=d(\mathbf{u}, \mathbf{v}) & =d\left(\mathbf{u}, \mathbf{z}_{2}\right)+d\left(\mathbf{z}_{2}, \mathbf{v}\right) \\
& \geqslant \Psi(E \cap[T])+\Psi A+2^{K-1}+1+\Psi B \\
& \geqslant \Psi(E \cap[T])+\frac{1}{2} \Psi[T+K+1]+2^{K-1} .
\end{aligned}
$$

D'autre part, on a $E=(E \cap[T]) \sqcup\left\{T, b_{1}, \ldots, b_{K}\right\}$ et $T \geqslant \Delta K$, donc

$$
\Psi E-\Psi(E \cap[T]) \leqslant \Psi[T+K+1]-\Psi[T]
$$

par le Lemme 2.7. Par conséquent

$$
D-\Psi E \geqslant \Psi[T]+2^{K-1}-\frac{1}{2} \Psi[T+K+1] .
$$

Or, cette dernière expression est positive, d'après (2.2).

Ceci termine la preuve du dernier cas, et le théorème est démontré. 


\section{Une conséquence du théorème}

Du Théorème 2.9 on déduit immédiatement le résultat annoncé en résumé de cet article, par une argumentation qui est classique [BH], mais que je rappelle ci-dessous.

Corollaire 4.1. Dans la variante à quatre colonnes des tours de Hanö̈, il faut au moins $\Phi(N)$ mouvements pour déplacer $N$ disques d'une colonne vers une autre.

Démonstration. On peut supposer $N \geqslant 1$. Soit $\mathbf{u}:[N] \rightarrow \mathcal{C}$ l'empilement où tous les disques sont dans la colonne 0 , et $\mathbf{v}$ celui où tous les disques sont dans la colonne 2. On définit le chemin $\gamma$ et les empilements intermédiaires $\mathbf{z}_{0}, \mathbf{z}_{2}$ comme précédemment; il les parcourt dans l'ordre suivant:

$$
\mathbf{u} \longrightarrow \mathbf{z}_{0} \longrightarrow \mathbf{z}_{2} \longrightarrow \mathbf{v}
$$

Dans l'empilement $\mathbf{z}_{0}^{\prime}$, la colonne 0 est vide ainsi qu'une autre; donc, par le Théorème 2.9,

$$
d\left(\mathbf{u}, \mathbf{z}_{0}\right) \geqslant d\left(\mathbf{u}^{\prime}, \mathbf{z}_{0}^{\prime}\right) \geqslant \Psi\{k \in[N-1]: \mathbf{u}(k)=0\}=\Psi[N-1] .
$$

De même, dans $\mathbf{z}_{2}^{\prime}$ la colonne 2 est vide ainsi qu'une autre; par ce même théorème,

$$
d\left(\mathbf{v}, \mathbf{z}_{2}\right) \geqslant d\left(\mathbf{v}^{\prime}, \mathbf{z}_{2}^{\prime}\right) \geqslant \Psi\{k \in[N-1]: \mathbf{v}(k)=2\}=\Psi[N-1] .
$$

Ici encore $d\left(\mathbf{z}_{0}, \mathbf{z}_{2}\right) \geqslant 1$, car le disque $N-1$ doit changer de colonne. Il en résulte que

$$
d(\mathbf{u}, \mathbf{v})=d\left(\mathbf{u}, \mathbf{z}_{0}\right)+d\left(\mathbf{z}_{0}, \mathbf{z}_{2}\right)+d\left(\mathbf{z}_{2}, \mathbf{v}\right) \geqslant 1+2 \Psi[N-1]=\Phi(N)
$$

par le Lemme 2.2, ce qu'il fallait démontrer.

\section{Remarques sur la démonstration}

Il faut maintenant expliquer comment je suis arrivé à cette preuve et en particulier, d'où vient cette fonction $\Psi$.

Dans le problème des Tours de Hanoï avec $N$ disques et $P$ tiges (avec $P \geqslant 3$ ), un rôle particulier est joué par les empilements critiques, c'est-à-dire ceux où au moins deux tiges sont vides. En effet, pour déplacer le plus grand disque d'une tige vers une autre, il est nécessaire (et suffisant) que ces deux tiges ne contiennent aucun autre disque, c'est-à-dire que les $N-1$ autres disques soient dans les $P-2$ colonnes restantes; autrement dit, que l'empilement restreint à $[N-1]$ soit critique.

Ces empilements critiques (après restriction) constituent donc des "checkpoints" dans le graphe des configurations $\mathrm{C}^{[N]}$ : tout chemin, géodésique ou non, entre deux empilements où la position du plus grand disque n'est pas la même, est obligé de traverser l'un d'eux. Pour dire les choses différemment, le graphe $\mathcal{C}^{[N]}$ présente une structure récursive: c'est une union de $P$ copies du graphe $\mathcal{C}^{[N-1]}$, 
et les arêtes joignant ces copies correspondent précisément aux empilements critiques de $N-1$ disques.

De ce point de vue, le problème classique des Tours de Hanoï, avec $P=3$, est essentiellement trivial: les empilements critiques ne sont autres que les empilements parfaits, où tous les disques sont dans une même colonne. Il y a donc seulement trois empilements critiques, un pour chaque colonne. En revanche, dans le problème de Dudeney, c'est-à-dire pour $P=4$, il y a $2^{N}$ configurations critiques pour chaque paire de colonnes. Ce très grand nombre constitue évidemment le cœur du problème.

La structure récursive du graphe suggère de procéder par récurrence sur le nombre de disques, $c^{\prime}$ est-à-dire de minorer certaines distances dans $\mathrm{C}^{[N]}$ en se basant sur les minorations analogues pour $N-1$ disques (ou moins).

Dans cette étude, on est constamment confronté au problème suivant: étant donné un empilement $\mathbf{u}$, et une colonne $c$, donner une minoration du nombre de mouvements nécessaires pour transférer tous les disques vers $P-2$ colonnes autres que $c$, qui ne dépende que du contenu de la colonne $c$ dans l'empilement de départ, c'est-à-dire de l'ensemble $\mathbf{u}^{-1}\{c\}$.

Pour les Tours de Hanoï classiques, i.e. $P=3$, le problème est facile: si $\mathbf{u}, \mathbf{v}$ : $[N] \rightarrow\{0,1,2\}$ sont deux empilements, avec $\mathbf{v}^{-1}\{2\}=[N]$, on a

$$
d(\mathbf{u}, \mathbf{v}) \geqslant \sum_{\substack{k \in[N] \\ \mathbf{u}(k)=0}} 2^{k}
$$

et le membre de droite ne dépend que de $\mathbf{u}^{-1}\{0\}$.

Un autre énoncé, dont on montre facilement l'équivalence avec le précédent, est celui-ci: pour $\mathbf{u}, \mathbf{v}:[N] \rightarrow\{0,1,2\}$ deux empilements arbitraires, on a

$$
d(\mathbf{u}, \mathbf{v}) \geqslant \sum_{k \in[N]} 2^{k}-\sum_{\substack{k \in[N] \\ \mathbf{u}(k) \neq 0}} 2^{k}-\sum_{\substack{k \in[N] \\ \mathbf{v}(k) \neq 2}} 2^{k}
$$

Dans le problème de Dudeney, i.e. $P=4$, la distance entre deux configurations parfaites est (conjecturalement) $\sum_{k \in[N]} 2^{\nabla k}$ au lieu de $\sum_{k \in[N]} 2^{k}$, c'est pourquoi il paraît naturel, par analogie avec la situation précédente, de proposer l'inégalité suivante: pour $\mathbf{u}, \mathbf{v}:[N] \rightarrow\{0,1,2,3\}$ deux empilements arbitraires, on a

$$
d(\mathbf{u}, \mathbf{v}) \geqslant \sum_{k \in[N]} 2^{\nabla k}-\sum_{\substack{k \in[N] \\ \mathbf{u}(k) \neq 0}} 2^{\nabla k}-\sum_{\substack{k \in[N] \\ \mathbf{v}(k) \neq 2}} 2^{\nabla k}
$$

En réalité, il existe d'autres arguments pour postuler une telle formule, plus convaincants que l'analogie formelle avec le problème à trois tiges, mais qui me mèneraient trop loin. Disons simplement qu'on sait calculer, moyennant la conjecture de Frame-Stewart, un certain nombre de distances dans le graphe $\mathfrak{C}^{[N]}$, pas seulement entre configurations parfaites; et que pour minorer toutes ces distances par une expression commune ne dépendant que de $\mathbf{u}^{-1}\{0\}$ et $\mathbf{v}^{-1}\{2\}$, la formule ci-dessus est essentiellement la seule raisonnable. 
Un autre énoncé, dont on montre facilement l'équivalence avec le précédent, est celui-ci: si $\mathbf{u}, \mathbf{v}:[N] \rightarrow\{0,1,2,3\}$ sont deux empilements avec $\mathbf{v}^{-1}\{0,1\}=\varnothing$, on a

$$
d(\mathbf{u}, \mathbf{v}) \geqslant \frac{\Phi(N+1)-1}{2}-\sum_{\substack{k \in[N] \\ \mathbf{u}(k) \neq 0}} 2^{\nabla k}
$$

C'est ce qu'on cherchait: une minoration (conjecturale) du nombre de mouvements nécessaires pour transférer tous les disques de $\mathbf{u}$ vers les colonnes 2 et 3 , qui ne dépend que de $\mathbf{u}^{-1}\{0\}$, et implique la conjecture de Frame-Stewart pour quatre tiges. Cette minoration présente également l'avantage d'être facilement réfutable par l'ordinateur. Et les calculs sur ordinateur, justement, ne font apparaître aucun contre-exemple pour les premières valeurs de $N$, ce qui est encourageant.

Il y a tout de même un problème, qui est que les inégalités (5.4) se prêtent mal à une démonstration par récurrence sur $N$. La raison est que ces inégalités, prises individuellement, sont parfois grossières; par exemple, le membre de droite peut être négatif.

On résout cette difficulté par "monotonisation" des minorants, c'est-à-dire en remplaçant cet ensemble d'inégalités par un autre ensemble d'inégalités qui sont collectivement équivalentes aux précédentes, mais individuellement plus fortes, en se basant sur une propriété de monotonie des distances.

De façon abstraite, cette procédure de monotonisation consiste juste à dire ceci: considérons deux fonctions $f, g: \mathcal{E} \rightarrow[0, \infty]$ sur un ensemble partiellement ordonné $\mathcal{E}$, qu'on souhaite comparer. La fonction $f$ est inconnue, mais on sait qu'elle est croissante, tandis que $g$ est connue, mais pas nécessairement croissante. Alors

$$
f \geqslant g \Longleftrightarrow f \geqslant \bar{g}
$$

où $\bar{g}$ est la plus petite fonction croissante qui majore $g$, i.e., $\bar{g}(x)=\sup _{y \leqslant x} g(y)$.

Pour les empilements sur $\mathcal{C}=\{0,1,2,3\}$, la situation est la suivante. Soient $\mathbf{u}, \mathbf{v}:[N] \rightarrow \mathcal{C}$ deux empilements de $N$ disques, $M$ un entier naturel $\leqslant N$, et $I:[M] \rightarrow[N]$ une fonction strictement croissante. Alors $\mathbf{u}^{\prime}=\mathbf{u} \circ I$ et $\mathbf{v}^{\prime}=\mathbf{v} \circ I$ sont deux empilements de $M$ disques, et il est manifeste que $d\left(\mathbf{u}^{\prime}, \mathbf{v}^{\prime}\right) \leqslant d(\mathbf{u}, \mathbf{v})$. Intuitivement, cela revient à sélectionner $M$ disques parmi les $N$, et ignorer les autres. On a déjà vu (et utilisé) cette propriété dans la démonstration du Théorème 2.9, dans le cas où $I$ est l'injection canonique de $[M]$ dans $[N]$.

Si $N$ est un entier naturel et $E$ une partie de $[N]$, définissons

$$
\Gamma(N, E)=\frac{\Phi(N+1)-1}{2}-\sum_{k \in[N]-E} 2^{\nabla k}
$$

Les inégalités (5.4) s'écrivent $d(\mathbf{u}, \mathbf{v}) \geqslant \Gamma\left(N, \mathbf{u}^{-1}\{0\}\right)$ si $\mathbf{v}^{-1}\{0,1\}=\varnothing$. En appliquant cette inégalité à $\mathbf{u} \circ I$ et $\mathbf{v} \circ I$ au lieu de $\mathbf{u}$ et $\mathbf{v}$, avec $I$ quelconque, on obtient

$$
d(\mathbf{u}, \mathbf{v}) \geqslant \operatorname{Sup}_{M \leqslant N} \operatorname{Sup}_{\substack{I:[M] \rightarrow[N] \\ \text { strictement } \\ \text { croissante }}} \Gamma\left(M, I^{-1} E\right)
$$


où $E=\mathbf{u}^{-1}\{0\}$, toujours sous l'hypothèse $\mathbf{v}^{-1}\{0,1\}=\varnothing$.

Voilà donc nos inégalités "monotonisées". Il ne reste plus qu'à calculer le membre de droite de (5.5). Le lecteur a déjà deviné la réponse: $c^{\prime} e s t \Psi(E)$.

Lemme 5.1. Soit $N$ un entier naturel, et E une partie de $[N]$. Pour tout entier naturel $M \leqslant N$ et toute fonction strictement croissante $I:[M] \rightarrow[N]$, on a $\Gamma\left(M, I^{-1} E\right) \leqslant \Psi E$. De plus, l'égalité est atteinte pour au moins un entier naturel $M \leqslant N$ et une fonction strictement croissante $I:[M] \rightarrow[N]$.

Démonstration. Soit $I:[M] \rightarrow[N]$ une fonction strictement croissante, avec $M \leqslant N$, et posons $B=I^{-1} E$. Par le Lemme 2.5, on a

$$
\Psi[M]-\Psi B \leqslant \sum_{k \in[M]-B} 2^{\nabla k}
$$

c'est-à-dire $\Gamma(M, B) \leqslant \Psi B$. Ensuite, on montre facilement que $\Psi B \leqslant \Psi(I B)$, avec les mêmes arguments que pour la deuxième inégalité du Lemme 2.4 (qui en est $\mathrm{d}$ 'ailleurs un cas particulier). Comme $I B \subseteq E$, ceci établit la première partie du lemme.

Montrons maintenant l'existence d'un cas d'égalité. Soit $L$ un entier naturel tel que $\Psi(E)=\Psi_{L}(E)$. Notons $K$ le nombre d'éléments $\geqslant \Delta(L+1)$ dans $E$, et soient $b(0)<\cdots<b(K-1)$ ces éléments. On a d'abord

$$
\Psi E=(1-L) 2^{L}-1+\sum_{k \in E} 2^{\min (\nabla k, L)}=(1-L) 2^{L}-1+K 2^{L}+\sum_{\substack{k \in E \\ k<\Delta(L+1)}} 2^{\nabla k}
$$

Définissons maintenant $M=\Delta(L+1)+K$, et $I:[M] \rightarrow[N]$ l'injection croissante définie par

$$
I(n)= \begin{cases}n & \text { si } n<\Delta(L+1), \\ b(n-\Delta(L+1)) & \text { sinon. }\end{cases}
$$

Pour cette fonction, on a

$$
\begin{aligned}
\Gamma\left(M, I^{-1} E\right) & =\Psi[M]-\sum_{\substack{k \in[M] \\
I(k) \notin E}} 2^{\nabla k}=\Psi[M]-\sum_{\substack{k<\Delta(L+1) \\
k \notin E}} 2^{\nabla k} \\
& =\Psi[M]-\Phi(\Delta(L+1))+\sum_{\substack{k<\Delta(L+1) \\
k \in E}} 2^{\nabla k}
\end{aligned}
$$

et par suite

$$
\begin{aligned}
\Gamma\left(M, I^{-1} E\right)-\Psi E & =\Psi[M]-\Phi(\Delta(L+1))-(1-L) 2^{L}+1-K 2^{L} \\
& =\Psi[M]-\Psi[\Delta(L+1)]-K 2^{L} \\
& =\sum_{\Delta(L+1)<k \leqslant M}\left(2^{(\nabla k)-1}-2^{L}\right) \geqslant 0
\end{aligned}
$$

ce qui montre l'égalité de $\Gamma\left(M, I^{-1} E\right)$ et $\Psi E$. 


\section{Références}

[BH] J.-P. BODE \& A. M. HINZ, Results and open problems on the Tower of Hanoi, Congressus Numerantium 139 (1999), 113-122

[CS] X. CHEN \& J. SHEN, On the Frame-Stewart conjecture about the Towers of Hanoi, SIAM Journal on Computing 33 (2004), 584-589

[Dud] H. E. DuDENEY, The Canterbury puzzles and other curious problems, E. P. Dutton, New York (1908)

[Dun] O. Dunkel, Editorial Note concerning Advanced Problem 3918, American Mathematical Monthly 48 (1941), page 219

$[\mathrm{H}+]$ A. M. Hinz, S. Klavžar, U. Milutinović \& C. Petr, The tower of Hanoi, myths and maths, Birkhaüser (2013)

[Fra] J. S. Frame, Solution to Advanced Problem 3918, American Mathematical Monthly 48 (1941), 216-217

[KF] R. E. KORF \& A. FELNER, Recent progress in heuristic search: a case study of the four-peg towers of Hanoi problem, Proceedings of the twentieth international joint conference on artificial intelligence, AAAI Press (2007), 23242329

[St1] B. M. StewART, Advanced Problem 3918, American Mathematical Monthly 46 (1939), page 363

[St2] B. M. SteWART, Solution to Advanced Problem 3918, American Mathematical Monthly 48 (1941), 217-219

[Sto] P. K. StOcKmeYeR, Variations on the four-post Tower of Hanoi puzzle, Congressus Numerantium 102 (1994), 3-12

[Sze] M. SzEGEDY, In how many steps the k-peg version of the Towers of Hanoi game can be solved?, Lecture Notes in Computer Science 1563 (1999), 356-361

Laboratoire de Mathématique (UMR 8628 du CNRS), bât. 425/430,

Université de Paris-Sud, 91405 Orsay Cedex, France.

E-mail: Thierry.Bousch@math.u-psud.fr 